\title{
A Mass Spectrometric System Equipped with Multi-Collectors and Peak Selectors for Continuous Gas Analysis
}

\author{
ISAO NISHI*
}

\begin{abstract}
A mass spectrometric system equipped with the multi-collectors and peak selectors is investigated. The principal features of this method are to make a rapid wide mass range scanning by the rapid voltage scanning of shorter range and to make a simultaneous recording of multi-components with fast response through the multi-channel peak selectors The graphic representation of ion optics is presented for determining the design parameters. Practical applications are given on the respiratory gas analysis.

Several technical key points such as determining the accurate collector position, the response of the system, and the characteristics of the gate circuit which is the central part of the peak selector, are discussed with the mind of applying this system to the respiratory gas analysis.
\end{abstract}

\section{Introduction}

In several fields, some problems require the continuous recording of the concentrations of gas mixtures in their changing phase. Particularly in the respiratory gas analysis, the strong desirability of the continuous and simultaneous recording of all the components of the respiratory gas has prompted the development of the mass spectrometer for such a use.

Early in 1949, Hunter et al. constructed the instrument equipped with three collectors positioned to accept $\mathrm{N}_{2}, \mathrm{O}_{2}$ and $\mathrm{CO}_{2}$." This type has been developed by many investigators. Almost all the instruments now available in the respiratory use belong to this type.

Another type for continuous analysis is the fast voltage scanning mass spectrometer first constructed for the respiratory use by Siri in 1947. ${ }^{2)}$ Fowler (1957) had systematically developed this type of mass spectrometer for the respiratory gas analysis to construct the commercially available instrument. ${ }^{3)}$ In his system, the respiratory gas peaks were displayed on C. R. T.; the top of the peaks was marked by the light spot. The movement of the spots was recorded photographically.

The more increase in a number of gases desirable to be detected, the more inconvenient becomes the multi-collector' system to be constructed. The voltage scanning mass spectrometer requires a larger scanning voltage with mass range spreading, and the time required for the individual beam to pass over the collector slit shortens at a required scanning frequency. This passing-over time becomes too short for the amplifier to make the accurate measurement of ion beam.

The foregoing considerations have led the author to devise a fast voltage scanning mass spectrometer equipped with the system composed of the multi-collectors and peak selectors in order to overcome the preceding defects.

In this paper the general description is given on the system developed by the author, and the graphical representation of ion optics for determining the design factors is presented with the applications to some practical problems.

* Faculty of Science and Technology, Tokyo University of Science, Noda, Chiba-ken, Japan 


\section{Nishi}

Several technical key points are discussed with the mind of applying this system to the respiratory gas analysis.

\section{General Description}

\subsection{Principle}

(1) In a given magnetic field, each mass has its focal point uniquely determined by the accelerating voltage and the scanning voltage can be replaced by the displacement of the collector position along the focal line.

(2) The ion beams accepted by a collector in sepuence can be spearated into the individual component through the peak selection circuits.

(3) When the sampling frequency is large enough, the analysis at intervals becomes effectively equal to the continuous analysis. These well-known facts are the principle of this method.

\subsection{General Lay-Out of the System}

The block diagram of the system is shown in Fig. 1. The system is composed of a voltage scanning sector type mass spectrometer equipped with the multi-collectors and peak selectors and of the direct gas inlet system. Each peak selector is composed of a voltage comparator, a monomulti-vibrator and a gate circuit with a peak-holder, or with a low-pass filter. When mass $M_{i}$ is fallen into collector $C_{1}$ by scanning, the gate for $M_{1}$ opens for a short time by the gate pulse generated in the monomulti-vibrator. The monomulti-vibrator is triggered by the signal from the voltage comparator. Trigger sign is generated just before $M_{1}$ appears in collector $C_{1}$ by comparing the scanning voltage with standard voltage peculiar to $M_{1}$. These series of circuits are prepared for individual masses. This mechanism of separation through the peak selector is illustrated schematically in Fig. 1.

Two types of the gate circuit tested are shown in Fig. 2. One is composed of the two analog switching circuits and a peak holder, the other of an analog switching circuit and a low-pass filter. Both are proved to be effectively used. The single F. E. T. can be substituted for the switching circuit.

Figure 3 shows the respiratory patterns in a prolonged breath measured by this system. These patterns are produced by eliminating variation factors from the original output of the system through the autormatic correction circuit.' The variation factors occur mainly in the direct sampling process. ${ }^{\text {) }}$

\section{Graphic Representation of Design Parameters on Ion Optics}

Figure 4 illustrates the schematical view of the ion trajectory which is to be discussed in this section. The shape of the magnetic field is taken rectangular.

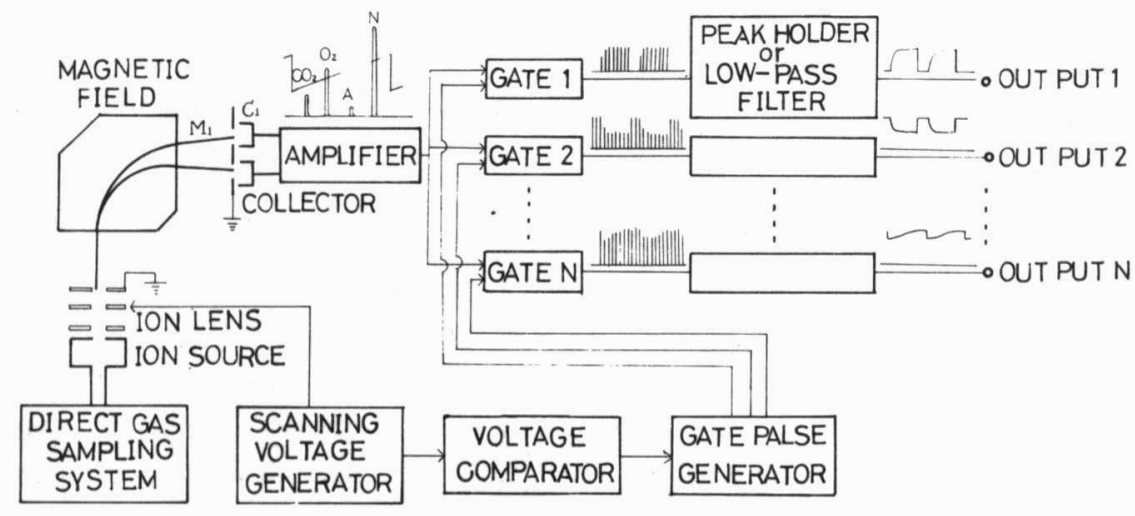

Fig. 1. Schematic diagram of the general lay-out. 


\section{A Mass Spectrometric System Equipped with Multi-Collectors and Peak Selectors for Continuous Gas Analysis}
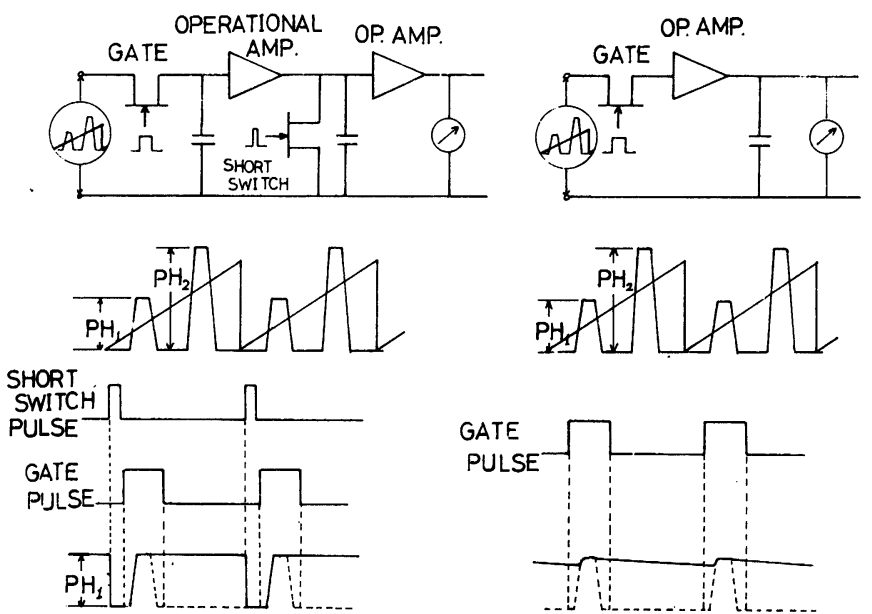

(a)

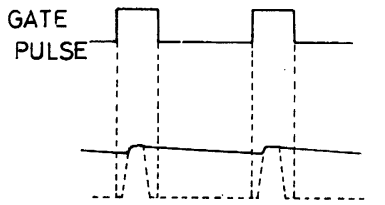

(b)

Fig. 2. Principle of the gate circuit: (a) with the peak holder, (b) with the low-pass filter.

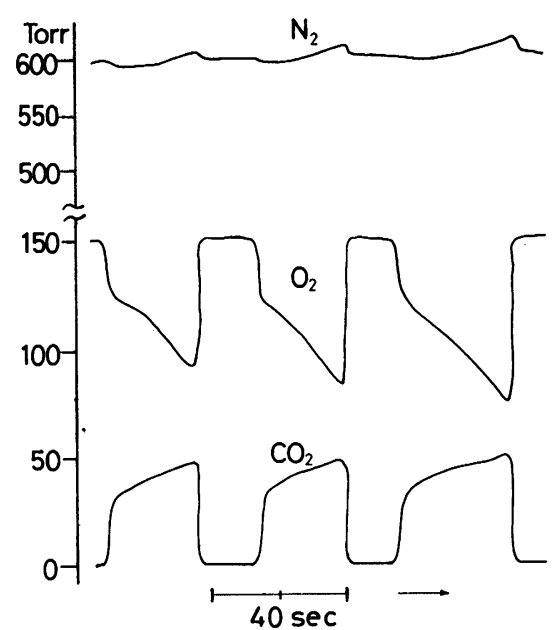

Fig. 3. Respiratory patterns measured by the system.

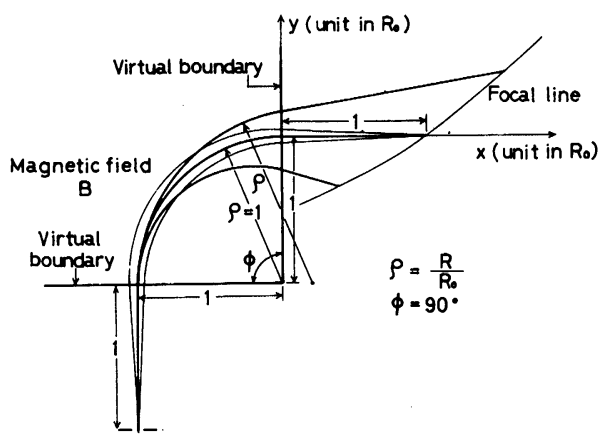

Fig. 4. Schematic view of the ion trajectory.
The graphic representation is deduced from the following well-known facts:

(1) The focal points of ion beams are determined by the extended Barbar's law.

(2) The action of ion motion in the homogeneous magnetic field is expressed as

$$
M=4.82 \times 10^{-5} \frac{R^{2} B^{2}}{V},
$$

where $M=$ mass of the singly charged ion in amu, $B=$ magnetic field intensity in gauss, $R=$ radius of the ion trajectory in $\mathrm{cm}$, and $V$ $=$ energy of the ion in volt.

Focal points $(x, y)$ are expressed as

$$
\begin{aligned}
& x=\frac{2 \rho-1}{\rho^{2}}(\rho \sqrt{2 \rho-1}+\rho-1) \\
& y=\sqrt{2 \rho-1}\left[1-\frac{1-\rho}{\rho} \sqrt{2 \rho-1}+\frac{(1-\rho)^{2}}{\rho^{2}}\right]-1
\end{aligned}
$$

according to Barbar's law, where $x$ and $y$ are the values of coordinates, and $\rho$ is the radius of the ion trajectory in the magnetic field.

They are scaled in $R_{0}$, the radius of the central 


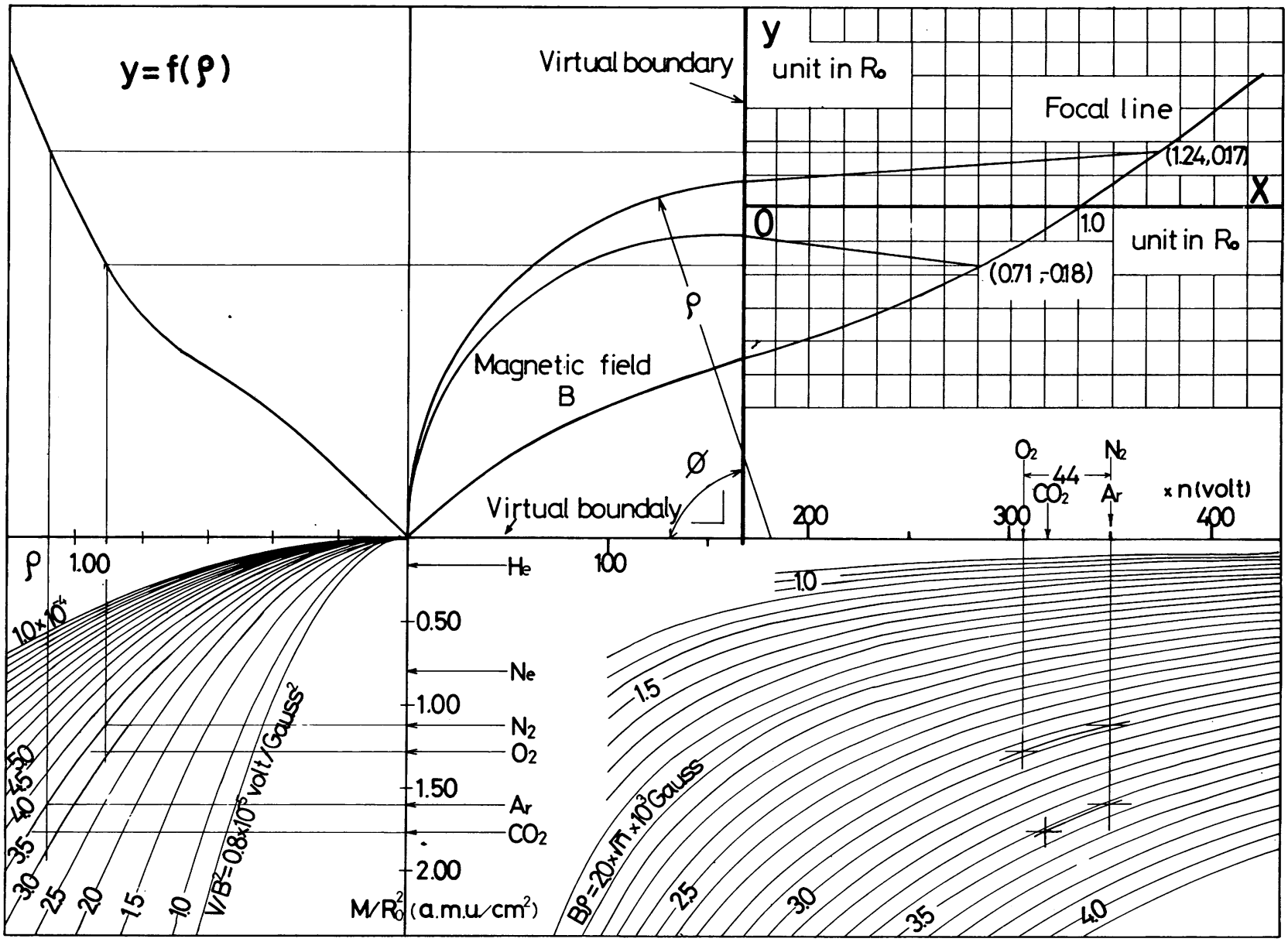

Fig. 5. Graphic representation of ion optics.

The real focal point $(X, Y)$ and the radius of the ion trajectory are expressed as $X=R_{0} x, Y=R_{0} y$ and $R=R_{0} \rho$. The accelerating voltage in the 4th quadrant is scaled in $n$ volt, where $n$ is the positive number. By using this expression, the representation becomes available for every range of the accelerating voltage. 


\section{A Mass Spectrometric System Equipped with Multi-Collectors and Peak Selectors for Continuous Gas Analysis}

ray in the symmetrical case. The results of Eqs. (2) and (3) are represented in the lst and 2nd quadrants in Fig. 5. In the figure, the real focal point $(X, Y)$ is expressed as $X=R_{0} x$ and $Y=R_{0} y$, and Eq. (1) is represented in the 3 rd and 4th quadrants.

It will be convenient to use this graphic representation for determining the specifications of the system. From the points on the particular line of $B^{2} / V$ constant in the 3rd quadrant of Fig. 5, the collector positions are . determined by the lining work; the components falling into the individual collectors are also simultaneously determined. The lining work is performed by the help of $y=\mathrm{f}(\rho)$ and the focal line.

When the magnetic field is given, the line of $B \rho$ in the 4th quadrant of Fig. 5 becomes the line of $\rho$. The points on the line of $\rho$ constant indicate how the marked gas components are accepted at a definite collector position by voltage scanning. When the accelerating voltage $V$ is given at a given magnetic field, the intersection of $V$ constant with the line of $B \rho$ constant also indicates the collector position and the components simultaneously falling into the collectors. This is helped by $y=\mathrm{f}(\rho)$ and the focal line.

In practical applications, the ion species to be detected are specified first; then the number of the collector, the collector position, the way of connecting the collectors, the magnetic field intensity, the accelerating voltage and its frequency, and the size and shape of the analyzer should be determined, considering the response of the measurement required, the characteristic of the amplifier for the ion detection, and the facilities of construction.

The width of the scanning voltage is determined by the lining work as illustrated in the 4th quadrant of Fig. 5.

\section{Practical Applications}

The practical applications of this method is investigated on the human breath analysis. In the respiratory work, the test gases as well as the physiological gases are to be detected. They are $\mathrm{He}(4 \mathrm{amu}), \mathrm{H}_{2} \mathrm{O}(18), \mathrm{Ne}(21), \mathrm{C}_{2} \mathrm{H}_{2}$ (26), $\mathrm{N}_{2}(28), \mathrm{O}_{2}(32), \operatorname{Ar}(40), \mathrm{CO}_{2}(44), \mathrm{N}_{2} \mathrm{O}$ (44, detectable on $\mathrm{NO}(30))$ and $\mathrm{SF}_{6}(146$, detectable on $F(19))$. In some applications, the concentrations of several components are required to measure simultaneously with the fast response of $0.1 \mathrm{sec}$ in the rise time.

Several examples are investigated in this section according to the graphic representation by the lining work. In this case the minimum accelerating voltage is taken about $300 \mathrm{~V}$. Our experiment shows that the beam width at the collector position is about $0.7 \mathrm{~mm}$ (ion source defining slit width is $0.4 \mathrm{~mm}$ ) at the accelerat ing voltage of $250 \mathrm{~V}$. It abruptly widens with the accelerating voltage lower than about $250 \mathrm{~V}$. ${ }^{*}$ The minimum accelerating voltage is preferable to facilitate the construction of the measuring system as long as the ion optical condition is satisfactory.

\section{Example 1}

As seen in Fig. 6, the system is composed of two collectors and two amplifiers for $\mathrm{N}_{2}$, $\mathrm{NO}$ (cracking pattern of $\mathrm{N}_{2} \mathrm{O}$ ), $\mathrm{O}_{2}, \mathrm{Ar}, \mathrm{CO}_{2}$ and $\mathrm{N}_{2} \mathrm{O}$. (Most of the respiratory work will be carried out by using this this system.) The lining for determining the design factors is illustrated in Fig. 5. The required resolving powers are 15 for $C_{1}$ and 10 for $C_{2}$.

\section{Example 2}

As seen in Fig. 7, this is the simplest system of all, which is composed of two collectors and an amplifier for $\mathrm{N}_{2}, \mathrm{O}_{2}, \mathrm{Ar}$ and

\footnotetext{
* The width is almost same with the higher accelerating voltage, while the peak height increases gradually to to the maximum value at the accelerating voltage of about $850 \mathrm{~V}$. These figures are seen in Figs. 10, 11 and 12, where the accelerating voltages for $\mathrm{O}_{2}$ are about $450 \mathrm{~V}, 550 \mathrm{~V}$ and $850 \mathrm{~V}$, respectively. (In the present case, the width of the defining slit is $0.4 \mathrm{~mm}$ and that of collector slits are $1.5 \mathrm{~mm}$ for $C_{1}$ and $2.0 \mathrm{~mm}$ for $C_{2}$.)
} 
I. Nishi

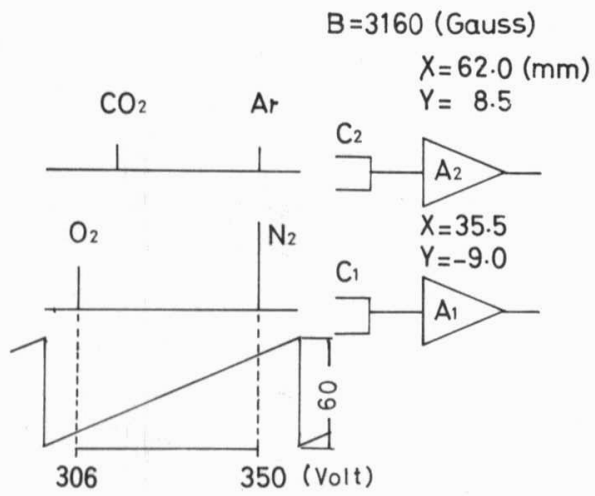

Fig. 6. Example 1. The real collector position $(X, Y)$ is expressed as $X=R_{0} x$ and $Y=R_{0} y, R_{0}=50 \mathrm{~mm}$ in the present case.

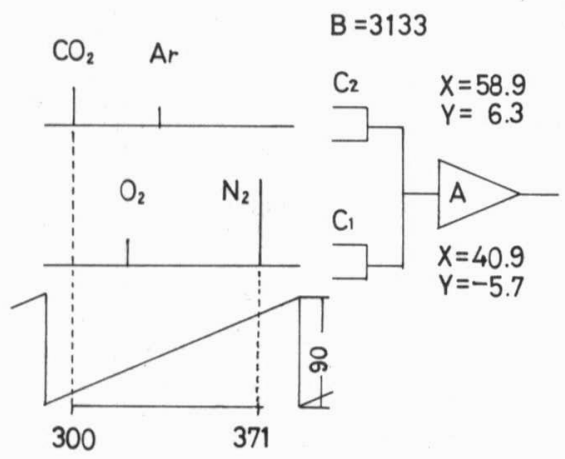

Fig. 7. Example 2.

$\mathrm{CO}_{2}$, The required resolving power is 15 .

\section{Example 3}

The system shown in Fig. 8 is composed of four collectors and two amplifiers for $\mathrm{He}$, $\mathrm{H}_{2} \mathrm{O}, \mathrm{F}$ (cracking pattern of $\mathrm{SF}_{6}$ ), $\mathrm{Ne}, \mathrm{C}_{2} \mathrm{H}_{2}$, $\mathrm{N}_{2}, \mathrm{NO}, \mathrm{O}_{2}, \mathrm{Ar}, \mathrm{CO}_{2}$ and $\mathrm{N}_{2} \mathrm{O}$. Almost all the applications on the respiratory work may be performed by this system. This system has the most stringent conditions positioning the collectors. The required resolving powers are 15 for $C_{1}-C_{2}$ system and 20 for $C_{2}-C_{4}$ system. $\mathrm{C}_{2}$ and $\mathrm{C}_{4}$ are located so as to accept $\mathrm{Ar}^{++}$ and $\mathrm{Ar}^{+}$at the same time. $\mathrm{C}_{1}$ and $\mathrm{C}_{3}$ are connected together and are located so as to accept $\mathrm{He}$ and $\mathrm{C}_{2} \mathrm{H}_{2}$ simultaneously. Such an arrangement is admitted because $\mathrm{He}$ and $\mathrm{C}_{2} \mathrm{H}_{2}$ are rarely used simultaneously in any respiratory work.

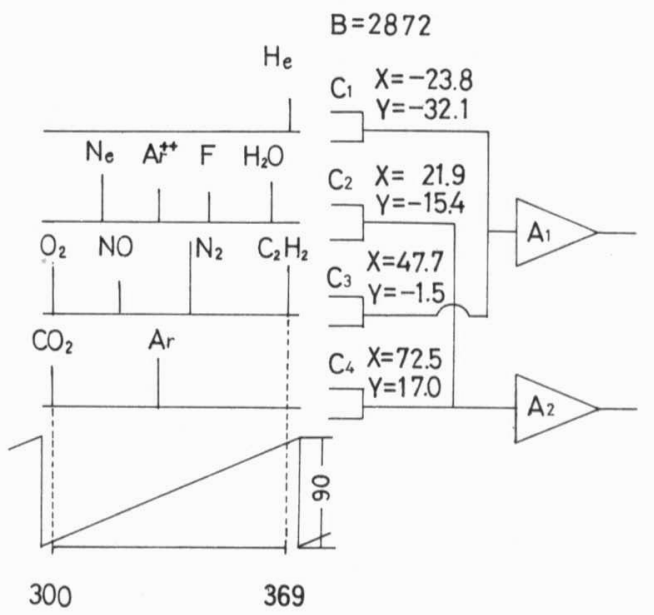

Fig. 8. Example 3.

\section{Discussion}

\subsection{Accuracy of Determining the Position}

Figure 9 shows the mass peaks of the mixture $\left(\mathrm{N}_{2}, \mathrm{O}_{2}, \mathrm{Ar}\right.$ and $\left.\mathrm{CO}_{2}\right)$ displayed on C. R. T. in the system of Example 1 described in the preceding section. In this case two collectors are to be located as to accept $\mathrm{N}_{2}$ and $\mathrm{Ar}$ at the same time. But a little displacement from the expected position was observed. This displacement is estimated to be about $0.4 \mathrm{~cm}$.

The examination of the fabrication of the collector system indicated that the discrepancy is mainly due to the poor setting collector positions in this case, and the deviation is $0.2 \mathrm{~mm}$ or more. However, the displacement

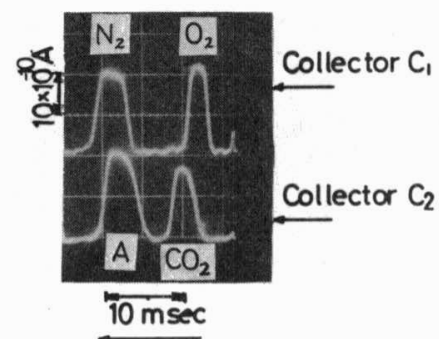

Fig. 9. Mass peaks accepted by collectors $C_{1}$ and $C_{2}$ in Example 1, the upper in $C_{1}$ and the lower in $C_{2}$. The peak widths are $2.2 \mathrm{~mm}$ for $C_{1}$ and $2.7 \mathrm{~mm}$ for $C_{2}$. The difference of the widths between $\mathrm{N}_{2}$ and $\mathrm{O}_{2}$ is due to the distortion of the scanning wave form. 


\section{A Mass Spectrometric System Equipped with Multi-Collectors and Peak Selectors for Continuous Gas Analysis}

of focal points occurs principally owing to the distortion of the distribution of the magnetic field from the ideal one. When the collectors are placed over the wide spread and the required resolving power is increased, this displacement becomes a stringent problem.

\subsection{Response}

In ordinary respiratory patterns, the builtup time from 0 to almost $100 \%$ is about $1 \mathrm{sec}$ as seen in Fig. 3. When the physiological dead space volume is determined for various gases from the rising curves of the respiratory patterns and the curves of the breathing volume, one percent accuracy is obtainable only when the sampling frequency is more than $100 / \mathrm{sec}$. The situation is the same for the detection of the cardiac ocillations. This means that the scanning frequency is desirable to be more than $100 \mathrm{~Hz}$ in the respiratory aplication.

Due to the limited band width of the amplifier, the detected peaks are distorted to the extent determined by the time constant of the detecting system and the scanning speed. Ezoe $^{6)}$ demonstrated in this calculation developed with an equivalent $R C$ integral circuit of the amplifier, how the top of the distorted output slides down the descending slope of the input and how the peak tails down to the follow ing peak, as $R C$ and the scanning speed increased. The distortion results in the shift of peaks as well as the reduction in the peak height, thus the second peak is affected by the preceding one.

According to his calculations, the following results are obtained for the trapezoidal peak as in the present case shown in Figs. 9, 10 and 11: The peak builds up to almost top $(99.5 \%)$ and falls down to almost zero in the time of $1.3 T$ for $C R / T=0.1$, where $T$ is the built-up time for $R C \rightarrow 0$, or the time required for the beam to pass over the width of the beam across the collector slit. These built-up and fall-down times are $1.5 T$ for $R C / T=0.15$.

In the present case, the time constant of the equivalent circuit is estimated to be 0.1 msec from the rise time $(10-90 \%)$ for the amplifier. (The rise time is $2.2 \times$ time constant.) The amplifier is composed of single operational amplifier and a feed-back resistance of $10^{9} \Omega$. Its rise time is about $0.2 \mathrm{sec}$ with the noise
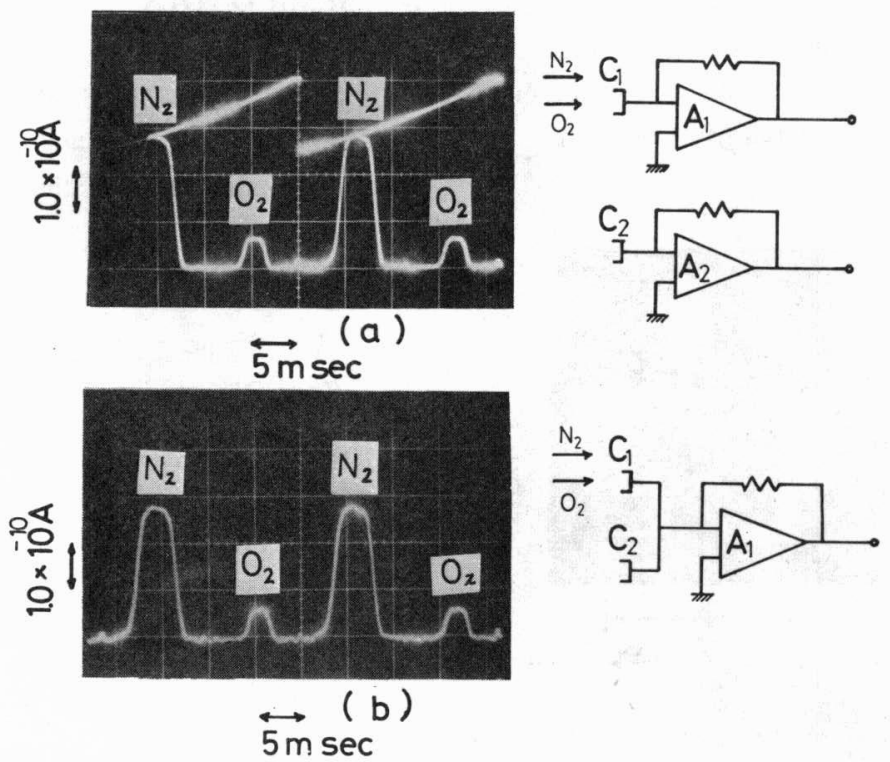

Fig. 10. Air spectra measured by different systems: (a) by the upper system, (b) by the lower system. 
level of $2 \times 10^{-12} \mathrm{~A}$, the built-up time $T=1.3$ msec for the scanning frequency of $50 \mathrm{~Hz}$ and $T=0.65 \mathrm{msec}$ for $100 \mathrm{~Hz}$ for the ideal sawingtooth wave scanning. Then $R C / T=0.1$ for $50 \mathrm{~Hz}$ and $R C / T=0.15$ for $100 \mathrm{~Hz}$. In a practical case as shown in Fig. 11, due to the distortion of the scanning wave, the rising times are not same for the same scanning frequency.

The permissible intervals between the adjacent peaks are $1.3 T+1.3 T=2.6 \times 1.3 \mathrm{msec}$ $=3.4 \mathrm{msec}$ for the scanning frequency of 50 $\mathrm{Hz}$ and $2.8 T=2.8 \times 0.65 \mathrm{msec}=1.8 \mathrm{msec}$ for $100 \mathrm{~Hz}$ when the overlapping of the peaks is avoided. On the other hand, the minimum designed intervals are estimated to be $10 \mathrm{msec}$, $5 \mathrm{msec}$ and $4 \mathrm{msec}$ in Examples 1, 2 and 3, respectively, for the scanning frequency of $50 \mathrm{~Hz}$, while the intervals for $100 \mathrm{~Hz}$ are $5 \mathrm{msec}$, $2.5 \mathrm{msec}$ and $2.0 \mathrm{msec}$ in Examples, 1, 2 and 3, respectively.

The preceding discussions indicate that $100 \mathrm{~Hz}$ in the scanning frequency is attainable in all examples with the simpler amplifier used in the present research. In practical applications of the respiratory use, however, 50-75 $\mathrm{Hz}$ in the scanning frequency is preferable, considering other various kinds of factors which affect the accuracy of the measurements, and the technical facility of the electronic system.

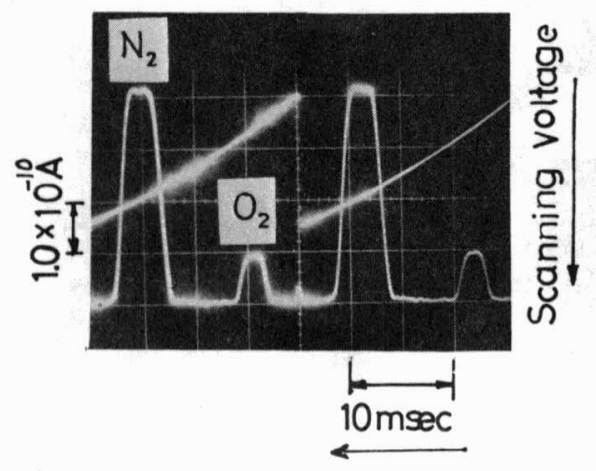

Fig. 11. Spectrum of the air in association with the scanning frequency. The width of the peak varies with the distortion of the scanning wave.
The difference of the response of two collector systems is examined on the air spectra displayed on C. R. T. The one spectrum is obtained through one collector-one amplifer system, and the other is obtained through two collectors-one amplifier system. These results are shown in Fig. 10 (a) and (b). The reduction of the response is hardly observed in sight with connecting two collectors. The connected collector system should be used with a possible care of reducing the input capcitance of the system.

\subsection{Leakage in Open Period}

The leakage of the input sine wave signal in the open period of point contact F. E. T. is shown in Fig. 12 with frequencies. The results indicate that when more than two ion beam fall into the same collector in sequence, the leakage of the largest one becomes the error of the smaller ones. When the peak height is $1 \%$ of the preceding one, using F. E. T. the error will be several percent in the case of Example 3 in which the peaks have the larger frequency components. With use of a micromodule type gate circuit such as TELEDYNE SEMICONDUCTOR 2126 BG hybrid microcircuit analog switch or $2128 \mathrm{BG}$, this leakage' is reduced by one order. This problem will be, however, completely resolved by using the gates in series connection.

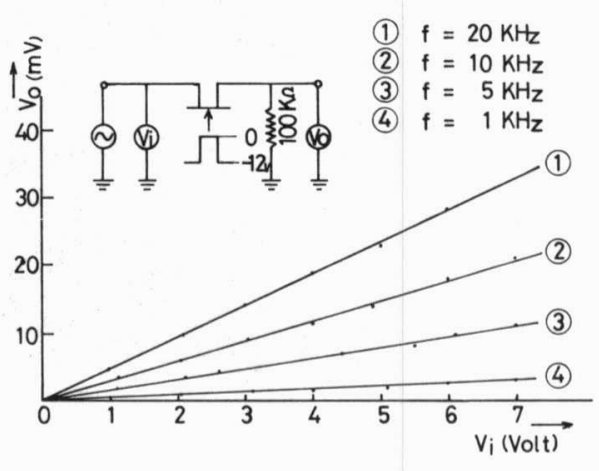

Fig. 12. Leakage of the input sine wave signal in the open period of point contact F. E. T. switching circuit. 


\section{A Mass Spectrometric System Equipped with Multi-Collectors and Peak Selectors for Continuous Gas Analysis}

\subsection{Noise Caused by Gate Pulse}

When the gate pulse is applied to the gate, a small signal is observed on the output terminal without input signal. This may be also the cause of the error. Figure 13 shows the step-up in the output when the gate pulse is applied to the switching circuit. This step-up voltage is less than about $0.5 \mathrm{mV}$ for the teledyne analog switch $2126 \mathrm{BG}$, which is almost neglegible in the respiratory application.

From the foregoing discussions, the mass spectrometric system developed in the present research is proved to be attainable to make a continuous recording of the concentrations of all the components of the respiratory gas in their changing phase, with the response of 0.1 $-0.2 \mathrm{sec}$ in the rise time. The accuracy in the simultaneous measurement is attainable to a few percent with the response of $0.1 \mathrm{sec}$ for the component of $1 \%$ in concentration by using the peak selector developed in the present research and the simple ion detector previously described. The largest ion beam can be taken as $1 \times 10^{-9} \mathrm{~A}$ and the smallest one is reampli-

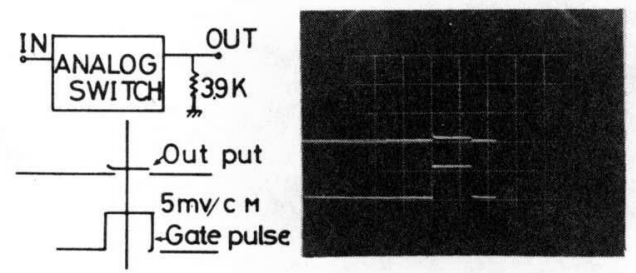

Fig. 13. Step-up voltage stimulated by the gate pulse on the switching circuit. fied by the second stage amplifier after separation, and the noise level of the first stage amplifier can be reduced to the value lower than $1 \times 10^{-13} \mathrm{~A}$ by filtering the high frequency components of the noise.

\section{Acknowledgments}

The author wishes to express his thanks to Mr. Sugai and Mr. Y. Shimizu for their help in the calculation. The assistance of $\mathrm{Mr}$. $\mathrm{K}$. Kimura in the experiment is also appreciated. The author is indebted to Mr. M. Kusakabe of the Toshiba Research and Development Center for his assistance in constructing the instrument, and to Mr. S. Inamoto, Assistant Professor of Electrical Engineering at Tokyo Lniversity of Science, for consulting on the switching circuit of electronic systems. The author would like to express his sincere gratitude to Dr. K. Kawasaki, Professor of Physics at Tokyo University of Science, for his constant encouragement in pursuing the present research.

\section{References}

1) J. A. Hunter, R. W. Stacy, and A. F. Hitchcock, Rev. Sci. Instr., 20, 333 (1949).

2) W. Siri, Rev. Sci. Instr., 18, 540 (1947).

3) K. T. Fowler, R. Hugh-Jones, British Medical J., 1, 1205 (1957).

4) I. Nishi, K. Kimura and S. Sugai, to be published in Mass Spectroscopy Japan.

5) I. Nishi, S. Sugai, and K. Kimura, to be published in the Proceedings of 2nd International Vacuum Conference.

6) H. Ezoe, Mass Spectroscopy Japan, 18, 966 (1970). 